\title{
Target Localization in Multipath Environment through the Exploitation of Multi-Frequency Array
}

\author{
Yimin D. Zhang, Xin Li, and Moeness G. Amin \\ Center for Advanced Communications \\ Villanova University, Villanova, PA 19085, USA \\ \{yimin.zhang, xin.1, moeness.amin\}@villanova.edu
}

\begin{abstract}
In this paper, we propose a multi-frequency array structure that achieves accurate target localization in a multipath environment. The use of multi-frequencies enables decorrelation in the array to operate direction-of-arrival (DOA) estimation for coherent arrival paths, whereas the spatial filtering of the signals makes the paths separable for robust range estimation. Multi-frequency radar techniques are then applied for range estimation of each path. The target position is identified by the shortest range coupled with its DOA.
\end{abstract}

\section{INTRODUCTION}

Target localization is generally an important task in many radar applications. One way to accurately localize a target is through the acquisition of combined range and direction-ofarrival (DOA) information. While wideband waveforms are desirable to obtain high-resolution range information, a very attractive alternative is to use a small number of continuous wave $(\mathrm{CW})$ sinusoids with different frequencies, provided that the targets are sparse. In particular, when only a single target is of interest, a dual-frequency can provide accurate range information [1, 2, 3]. As a result, an array exploiting dualfrequency signaling is shown to be effective for the target localization [4]. The use of more frequencies may be beneficial to achieve frequency diversity and/or to achieve larger unambiguous range estimations $[5,6]$.

The use of sparse waveforms, on the other hand, is sensitive to various factors. One critical problem in multifrequency radar system is the multipath propagation. For a moving target, when its return signal and the multipath replicas demonstrate different Doppler signatures, the multipath signals become separable in the frequency or timefrequency domain and proper range estimation becomes possible [3]. When a target is stationary or slowly timevarying, range estimation in a multipath environment remains a challenging problem. Coherent multipaths also destroy the full rank requirement necessary for DOA estimation, making the target localization problem very difficult.

In this paper, we propose a multi-frequency array structure that achieves accurate target localization in such a multipath environment. The use of multi-frequency enables decorrelation in the array to operate DOA estimation for coherent arrival paths, whereas the spatial filtering of the signals makes the paths separable for robust range estimation. Multi-frequency radar techniques are then applied for range estimation of each path. The target position is identified by the shortest range coupled with its DOA.

It is noted that a similar problem was considered in the context of radio frequency identification (RFID) system for a two-path model [7]. However, the signal model considered therein does not include the cross-paths (propagating forward through direct path and returning back from reflection path, or vice versa). The inclusion of the cross-path will alter the phase information, rendering inaccurate range and DOA estimations. While this work is developed upon the same spirit, the contribution lies in the consideration of a general problem exploiting multi-antenna multi-frequency radar with the inclusion of the cross-path propagations. A scheme is developed for accurate target localization when the cross-path is properly taken into account. The target localizability is also considered in this paper.

\section{Signal Model}

\section{A. Signal Model}

Consider an $N$-element array $(N \geq 3)$ as illustrated in Fig. 1 . Without loss of generality, we denote antenna 1 as the reference antenna located at the origin, from where a waveform consisting of $M$-frequency CWs $(M \geq 3)$,

$$
s(t)=\sum_{i=1}^{M} \exp \left(j 2 \pi f_{i} t\right)
$$

is transmitted. The signal arrival at the target position becomes

$$
\begin{aligned}
x(t) & =\sum_{q=1}^{Q} \sum_{i=1}^{M} h_{q}\left(f_{i}\right) \exp \left(j 2 \pi f_{i} t-j 2 \pi f_{i} R_{q} / c\right) \\
& =\sum_{q=1}^{Q} \sum_{i=1}^{M} h_{q}\left(f_{i}\right) \exp \left(j 2 \pi f_{i} t-j k_{i} R_{q}\right),
\end{aligned}
$$

where $Q \geq 2$ is the number of multipaths, $R_{q}$ is the overall path length of path $q, h_{q}\left(f_{i}\right)$ is the corresponding path coefficient at frequency $f_{i}, c$ is the propagation velocity, and $k_{i}=2 \pi f_{i} / c$. We use $q=1$ to identify the direct path and $q>1$ refers to the reflection path. Coefficients $h_{1}\left(f_{i}\right)$ take a real value as they represent the path loss, whereas $h_{q}\left(f_{i}\right)$ for $q>1$ may take complex value in general as the result of possible phase change in the course of reflection. It is reasonably assumed that $h_{q}\left(f_{i}\right)$ for $q>1$ may take necessarily the same value for the $M$ frequencies but their value should remain close. 


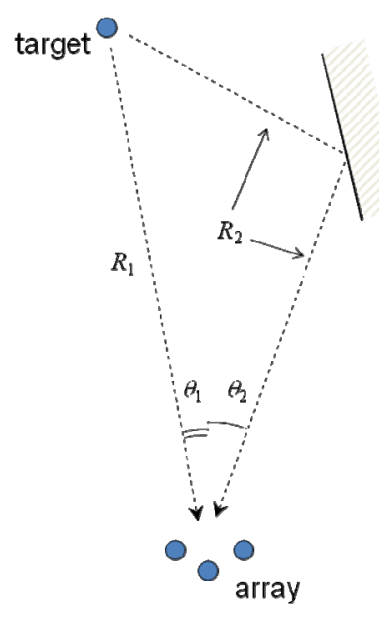

Figure 1. Example of multipath scenario.

The backscattered signal vector received at the array is expressed as

$$
\begin{aligned}
\mathbf{r}(t) & =\rho \sum_{q=1}^{Q} \mathbf{a}_{q} h_{q} x(t) \exp \left(-j k_{i} R_{q}\right) \\
& =\rho \sum_{q=1}^{Q} \sum_{p=1}^{Q} \sum_{i=1}^{M} \mathbf{a}_{q}\left(f_{i}\right) h_{q}\left(f_{i}\right) h_{p}\left(f_{i}\right) \exp \left[j 2 \pi f_{i} t-j k_{i}\left(R_{p}+R_{q}\right)\right],
\end{aligned}
$$

where $\rho$ is the target reflection coefficient,

$$
\mathbf{a}_{q}\left(f_{i}\right)=\left[\begin{array}{llll}
1 & \exp \left(-j k_{i} \mathbf{z}_{2}^{T} \mathbf{l}_{q}\right) & \cdots & \exp \left(-j k_{i} \mathbf{z}_{N}^{T} \mathbf{l}_{q}\right)
\end{array}\right]^{T}
$$

is the steering vector corresponding to DOA $\theta_{q}$ and frequency $f_{i}$, with $\mathbf{z}_{n}$ denoting the location vector of the $n$th antenna and ${ }^{T}$ denoting the transpose of a matrix or a vector, and $\mathbf{I}_{q}$ is the unit vector in the direction of $\theta_{q}$. We assume that $\rho$ in general takes a complex value but is considered frequencyindependent for the small frequency separations being considered. As a result, $\rho$ is an irrelevant term in phase difference-based DOA and range estimation and thus is omitted hereafter for the succinctness of presentation.

Filtering the $M$ frequency components of $\mathbf{r}(t)$ using corresponding filters and downconverting the results to baseband yields the following $M$ separated vector outputs,

$$
\mathbf{y}_{i}(t)=\sum_{q=1}^{Q} \sum_{p=1}^{Q} \mathbf{a}_{q}\left(f_{i}\right) h_{q}\left(f_{i}\right) h_{p}\left(f_{i}\right) \exp \left[-j k_{i}\left(R_{p}+R_{q}\right)\right]+\mathbf{n}_{i}(t),
$$

where $\mathbf{n}_{i}(t)$ is the additive noise vector.

\section{TARGET LOCALIZATION}

Target localization is achieved through the combined use of the DOA and range information of the direct path. The general processing flowchart is depicted in Fig. 2, and the detailed signal processing procedures will be addressed below.

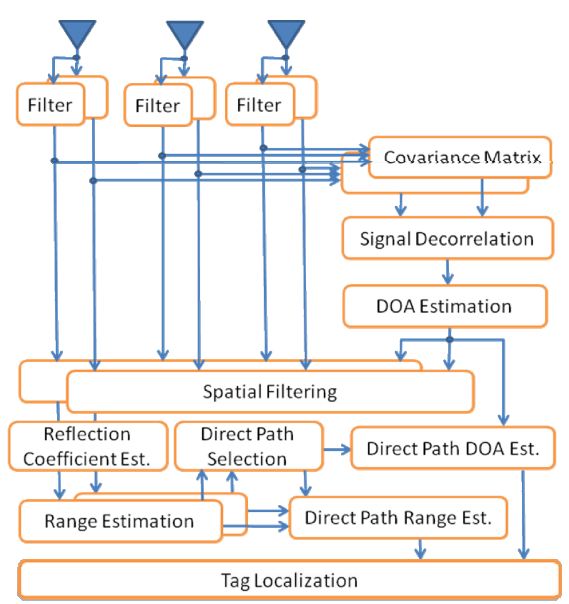

Figure 2. Flowchart of the proposed localization technique.

\section{A. DOA Estimation}

DOA estimation in each carrier frequency is impractical because the signal arrivals corresponding to different paths are coherent for the narrowband waveforms we are using. Spatial smoothing techniques [8] can be used for signal decorrelation, but only at the cost of reduced array degreesof-freedom (in other words, more antennas would become necessary). This is highly infeasible when dealing with an array consisting of a small number of elements.

In this work, the decorrelation is achieved through the averaging the covariance matrices obtained from different frequencies. The approach is very similar to what presented in [9], but we are dealing with a multi-frequency signal instead of a wideband signal. For the convenience of formulation, we introduce the baseband equivalence of the $M$ frequency components in $x(t)$ as

$$
\widetilde{x}_{i}=\sum_{q=1}^{Q} h_{q}\left(f_{i}\right) \exp \left(-j k_{i} R_{q}\right)
$$

Thus, $\mathbf{y}_{i}(t)$ can be rewritten as

$$
\mathbf{y}_{i}(t)=\sum_{q=1}^{Q} \mathbf{a}_{q}\left(f_{i}\right) h_{q}\left(f_{i}\right) \widetilde{x}_{i} \exp \left(-j k_{i} R_{q}\right)=\rho \mathbf{A}\left(f_{i}\right) \widetilde{x}_{i} \mathbf{h}\left(f_{i}\right)+\mathbf{n}_{i}(t),
$$

where $\mathbf{A}\left(f_{i}\right)=\left[\mathbf{a}_{1}\left(f_{i}\right) \cdots \mathbf{a}_{Q}\left(f_{i}\right)\right]$ is the steering matrix, and $\mathbf{h}\left(f_{i}\right)=\left[\begin{array}{llll}h_{1}\left(f_{i}\right) \exp \left(-j k_{i} R_{1}\right) & \cdots & h_{Q}\left(f_{i}\right) \exp \left(-j k_{i} R_{Q}\right)\end{array}\right]^{T}$.

To fuse the information observed at the $M$ different frequencies, the use of linear transformation of the signal subspace at each frequency into a reference frequency is well known $[9,10,11]$. Denote $\mathbf{T}_{i}$ as the focusing matrix to transform the signal subspace at frequency $f_{i}$ into a signal subspace at frequency $f_{0}$, i.e., $\mathbf{T}_{i} \mathbf{A}\left(f_{i}\right)=\mathbf{A}\left(f_{0}\right)$. In practice, $f_{0}$ is chosen within the range of frequencies being used. By applying the focusing matrix into (6), we obtain 


$$
\tilde{\mathbf{y}}_{i}(t)=\mathbf{T}_{i} \mathbf{y}_{i}(t)=\mathbf{A}\left(f_{0}\right) \tilde{x}_{i} \mathbf{h}\left(f_{i}\right)+\tilde{\mathbf{n}}_{i}(t) .
$$

The covariance matrix of the above transformed signal vector is expressed as

$$
\widetilde{\mathbf{R}}_{i}=E\left[\widetilde{\mathbf{y}}_{i}(t) \widetilde{\mathbf{y}}_{i}^{H}(t)\right]=\left|\widetilde{x}_{i}\right|^{2} \mathbf{A}\left(f_{0}\right) \mathbf{h}\left(f_{i}\right) \mathbf{h}^{H}\left(f_{i}\right) \mathbf{A}^{H}\left(f_{0}\right)+\sigma_{n}^{2} \mathbf{I}_{L},
$$

where ${ }^{H}$ denotes conjugate transpose, $\sigma_{n}^{2}$ is the noise variance, and $\mathbf{I}_{L}$ is the $L \times L$ identity matrix. Averaging $\widetilde{\mathbf{R}}_{i}$ over the $M$ frequencies yields

$\overline{\mathbf{R}}=\frac{1}{M} \sum_{i=1}^{M} \widetilde{\mathbf{R}}_{i}=\mathbf{A}\left(f_{0}\right)\left[\frac{1}{M} \sum_{i=1}^{M}\left|\widetilde{x}_{i}\right|^{2} \mathbf{h}\left(f_{i}\right) \mathbf{h}^{H}\left(f_{i}\right)\right] \mathbf{A}^{H}\left(f_{0}\right)$.

When $\exp \left(-j k_{i} R_{q}\right)$ takes unequal values for $i=1, \ldots, M$ and $q=1, \ldots, Q$, the rank of matrix $\overline{\mathbf{H}}=\frac{1}{M} \sum_{i=1}^{M}\left|\widetilde{x}_{i}\right|^{2} \mathbf{h}\left(f_{i}\right) \mathbf{h}^{H}\left(f_{i}\right)$ is $\min (Q, M)$. Therefore, the DOA information of the $Q$ paths can be estimated, provided that $M \geq Q$ is satisfied. Apparently, large separations in frequency and path length will yield better decorrelation for rank restoration. However, the frequency separation is bounded by the unambiguous range issue, whereas the path length difference is determined by the propagation environment.

DOA estimation can be, for example, performed using the MUSIC algorithm by finding the $Q$ peaks of the following pseudo-spectrum

$$
F=\left\|\mathbf{a}^{H}(\theta) \mathbf{U}\right\|^{-2},
$$

where $\mathbf{a}(\theta)$ is the steering vector corresponding to DOA $\theta$, and $\mathbf{U}$ is a $L \times(L-Q)$ matrix (or vector if $L-Q=1$ ) spanning the noise subspace of $\overline{\mathbf{R}}$.

It is note that, in many applications, the frequency separation is usually much less than the carrier frequencies to meet the unambiguous range requirement. In this case, the operation of focusing matrix projection is optional because omitting such operation only results in insignificant error in DOA estimations.

\section{B. Spatial Filtering}

Before range estimation can be made for each path, we need to perform spatial filtering to separate each signals by projecting the received signal vector into the null subspace of other paths. The projection matrix to retrieve the frequency $f_{i}$ component of the received signal corresponding to path $p$ is expressed as

$$
\mathbf{P}_{p}^{\perp}\left(f_{i}\right)=\mathbf{I}_{N}-\hat{\mathbf{A}}_{\bar{p}}\left(f_{i}\right)\left[\hat{\mathbf{A}}_{\bar{p}}^{H}\left(f_{i}\right) \hat{\mathbf{A}}_{\bar{p}}\left(f_{i}\right)\right]^{-1} \hat{\mathbf{A}}_{\bar{p}}^{H}\left(f_{i}\right),
$$

where $\hat{\mathbf{A}}_{\bar{p}}\left(f_{i}\right)$ is the resulting matrix by removing the $p$ th column from $\hat{\mathbf{A}}\left(f_{i}\right)$, an estimate of $\mathbf{A}\left(f_{i}\right)$ constructed from the estimated DOA information. Note that we use $p$, instead of $q$, as the index of path to emphasize the fact that we do not have the knowledge which path is the direct path. To avoid the consideration of scaling in the projection, we use the following oblique projection matrix

$$
\mathbf{P}_{p}\left(f_{i}\right)=\mathbf{a}_{p}\left(f_{i}\right)\left[\mathbf{a}_{p}^{H}\left(f_{i}\right) \mathbf{P}_{p}^{\perp}\left(f_{i}\right) \mathbf{a}_{p}\left(f_{i}\right)\right]^{-1} \mathbf{a}_{p}^{H}\left(f_{i}\right) \mathbf{P}_{p}^{\perp}\left(f_{i}\right) .
$$

The separated signal vector for path $p$ at frequency $f_{i}$ is expressed as

$$
\mathbf{z}_{i, p}(t)=\mathbf{P}_{p}\left(f_{i}\right) \mathbf{y}_{i}(t)=\mathbf{P}_{p}\left[\mathbf{A}\left(f_{i}\right) \tilde{x}_{i} \mathbf{h}\left(f_{i}\right)+\mathbf{n}_{i}(t)\right] .
$$

Ideally, when $\hat{\mathbf{A}}\left(f_{i}\right)=\mathbf{A}\left(f_{i}\right)$, only the signal received from the $p$ th path will be retrieved at $\mathbf{z}_{i, p}(t)$, i.e.,

$$
\mathbf{z}_{i, p}(t)=\mathbf{a}_{p}\left(f_{i}\right) \tilde{x}_{i} h_{p}\left(f_{i}\right) \exp \left(-j k_{i} R_{p}\right)+\mathbf{n}_{i, p}(t) .
$$

When there is estimation error in $\hat{\mathbf{A}}\left(f_{i}\right)$, however, $\mathbf{z}_{i, p}(t)$ will carry such error as well, yielding error propagation in the range estimation step as described below.

\section{Range Estimation}

Noting the fact that $\mathbf{a}_{p}^{H}\left(f_{i}\right) \mathbf{a}_{p}\left(f_{i}\right)=L$, left multiplying $\mathbf{z}_{i, p}(t)$ with $(1 / L) \mathbf{a}_{p}^{H}\left(f_{i}\right)$ and taking average over the available observation time yields

$$
\widetilde{z}_{i, p}=\left\langle\frac{1}{L} \mathbf{a}_{p}^{H}\left(f_{i}\right) \mathbf{z}_{i, p}(t)\right\rangle=\widetilde{x}_{i} h_{p}\left(f_{i}\right) \exp \left(-j k_{i} R_{p}\right)+\tilde{n}_{i, p} .
$$

where $\widetilde{n}_{i, p}(t)=\left\langle\frac{1}{L} \mathbf{a}_{p}^{H}\left(f_{i}\right) \mathbf{n}_{i, p}(t)\right\rangle$ with $\langle\cdot\rangle$ denoting time average. Because $h_{1}$ is real, the phase information contained in the $\exp \left(-j k_{i} R_{p}\right)$ term can be used to recover the range $R_{p}$ through dual- or multi-frequency radar techniques once $\tilde{x}_{i}$ is estimated. From (5) and (14), we find that

$$
\sum_{p=1}^{Q} \widetilde{z}_{i, p}=\widetilde{x}_{i} \sum_{p=1}^{Q} h_{p}\left(f_{i}\right) \exp \left(-j k_{i} R_{p}\right)+\sum_{p=1}^{Q} \widetilde{n}_{i, p}=\widetilde{x}_{i}^{2}+\sum_{p=1}^{Q} \widetilde{n}_{i, p} .
$$

Because the last term is zero mean Gaussian noise, $\tilde{x}_{i}$ is estimated as

$$
\tilde{x}_{i}= \pm\left[\sum_{p=1}^{Q} \widetilde{z}_{i, p}\right]^{\frac{1}{2}} .
$$

Note that the ambiguity in the sign of $\tilde{x}_{i}$ estimation yields additional phase ambiguity, as discussed below.

Assuming that $\tilde{x}_{i}$ is accurately estimated and its effect is removed from (14), we obtain

$$
\widetilde{z}_{i, p}^{\prime}=h_{p}\left(f_{i}\right) \exp \left(-j k_{i} R_{p}\right)+\tilde{x}_{i}^{-1} \widetilde{n}_{i, p} .
$$

In the following, we summarize the multi-frequency radar techniques for range estimation based on the above result $[5$, 6]. 
If we do not consider the phase wrapping in the received signal, range $R_{p}$ can be estimated from the phase difference observed at the return signal corresponding to any pair of the operating frequencies, i.e.,

$$
R_{p}=\frac{\varphi_{i, p}-\varphi_{j, p}}{k_{i}-k_{j}}=\frac{c}{2 \pi} \frac{\varphi_{i, p}-\varphi_{j, p}}{f_{i}-f_{j}},
$$

where $\varphi_{i, p}=-\angle \widetilde{z}^{\prime}{ }_{i, p}(t)$. In reality, however, phase observations are wrapped within the $[0 ; 2 \pi)$ range and, due to the sign ambiguity in (18), the true phase can be expressed as

$$
\varphi_{p}^{(\text {true })}=\varphi_{i, p}-\varphi_{j, p}+\pi m_{p}^{(i, j)}
$$

where $m_{p}^{(i, j)}$ is an unknown integer. Accordingly, the range estimate is subject to range ambiguity $[1,2,3]$, i.e.,

$$
R_{p}=\frac{c}{2 \pi} \frac{\varphi_{i, p}-\varphi_{j, p}}{f_{i}-f_{j}}+\frac{c m_{p}^{(i, j)}}{2\left(f_{i}-f_{j}\right)} .
$$

The second term in the above equation reflects uncertainty in the tag range. For the same phase difference, the range can assume infinite values separated by the following maximum unambiguous range determined by the frequency separation,

$$
R_{\max }^{(i, j)}=\frac{c m_{p}^{(i, j)}}{2\left(f_{i}-f_{j}\right)} .
$$

It is evident from (23) that $R_{\max }^{(i, j)}$ depends on the difference between the two frequencies $f_{i}$ and $f_{k}$. There generally exists a tradeoff between the unambiguous range and the sensitivity of range estimates to noise. When more than two frequencies are available, we can choose unequal frequency differences between the adjacent pairs to yield a much longer overall unambiguous range than that of each individual pair [6]. A sophisticated scheme for frequency selection with unequal frequency differences was developed based on the Chinese remainder theorem in [5].

Once the ranges of all the paths are obtained, the shortest path is considered as the direct path. The paring of the range information with the corresponding DOA information will yield the localization of the target.

\section{Simulation Results}

In this section, we provide a number of simulation results to demonstrate performance of the proposed scheme. We use a three-element uniform linear array with element spacing of $0.16 \mathrm{~m}$, which is half-wavelength at $f=925 \mathrm{MHz}$. Three carrier frequencies of 905,914 and $925 \mathrm{MHz}$ are considered, unless otherwise specified. The target is located at a range of $5 \mathrm{~m}$ from the center of the array, and the DOA of the direct path is $\theta_{1}=-15^{\circ}$. The reflection path with a total length of $R_{2}$ $=7 \mathrm{~m}$ is assumed, and the DOA of the reflection point is $\theta_{2}=20^{\circ}$. The reflection coefficient is assumed to be $h_{2}=0.7 e^{j \pi / 4}$. The reflection coefficient is unknown to the system and has to be estimated. In each data set, 100 data samples are used for processing. The average input signal-tonoise ratio (SNR) at each sample is set to $6 \mathrm{~dB}$. In the underlying application, the relative frequency separation is sufficiently small and thus the focusing operation is not performed.

The estimated target positions are illustrated in Figure 3, where two frequency sets are respectively considered. The simulated results from 100 independent trials are depicted in each plot. The actual target position is marked as a red circle for reference. For frequency set I, the maximum frequency separation is $20 \mathrm{MHz}$, whereas it reduces to $10 \mathrm{MHz}$ for frequency set II. The localization accuracy degrades as the frequency separation reduces because the phase difference under a smaller frequency separation is less effective for the decorrelation of two coherent paths and the phase difference is more vulnerable to noise. For example, the mean square error (MSE) of the estimated target position increases from $0.084 \mathrm{~m}$ for frequency set I to $0.228 \mathrm{~m}$ for frequency set II. In the following simulation examples, therefore, frequency set I is used.

In the two plots depicted in Figure 4, the DOA of the reflection path is respectively changed to $40^{\circ}$ and $10^{\circ}$, whereas the DOA of the direct path remains to be $-15^{\circ}$. Compared to Figure 3(a), where the DOA of the reflection path is $20^{\circ}$, the MSE of the estimation position is slightly improved to $0.066 \mathrm{~m}$ for $\theta_{2}=40^{\circ}$, whereas it degrades to $0.141 \mathrm{~m}$ when $\theta_{2}=10^{\circ}$ is assumed. A wider separation of paths yields improved DOA estimations and more effective spatial filtering of the paths.

In Figure 5, we change the length of the reflection path from $7 \mathrm{~m}$, as used in Figure 3, to $R_{2}=6 \mathrm{~m}$ and $R_{2}=8 \mathrm{~m}$, respectively. The other parameters remain the same as those used in Figure 3(a). As a result, the MSE degrades to $0.181 \mathrm{~m}$ for the $R_{2}=6 \mathrm{~m}$ case and improves to $0.067 \mathrm{~m}$ for the $R_{2}=8 \mathrm{~m}$ case. The primary reason behind this is that a larger path difference between the direct and reflection paths will likely increase the phase difference for effective signal decorrelation. It is also mentioned that the change of reflection path length may also alter the strength of the mixed signal at the target, yielding variations in the target localization performance.

Similarly, the amplitude and phase of the reflection coefficient $h_{2}$ of the reflection path can also change the strength of the mixed signal at the target. To demonstrate this, Figure 6 illustrates the localization results where reflection coefficient is set to $h_{2}=0.3 e^{j \pi / 4}$ and $h_{2}=0.7 e^{-j \pi / 4}$, respectively. Compared to Figure 3(a), the MSE degrades to 0.1352 $\mathrm{m}$ in Figure 6(a) as the amplitude of the reflection coefficient is reduced to 0.3, whereas in Figure 6(b) the MSE degrades to $0.149 \mathrm{~m}$ as the magnitude of the reflection coefficient is maintained to 0.7 and the phase of reflection coefficient is reversed. 


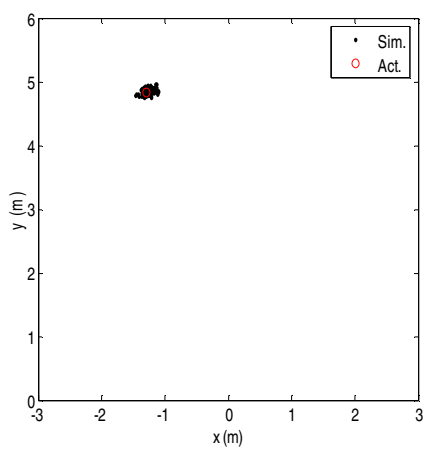

(a) Frequency set I

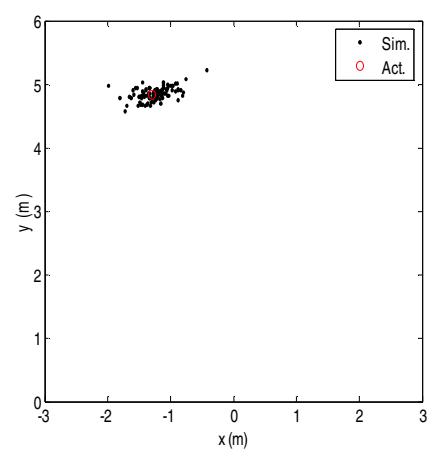

(b) Frequency set II
Figure 3. Estimated target positions using two frequency sets: (a) $[905,914,925] \mathrm{MHz}$ and (b) $[905,909,915] \mathrm{MHz}$.

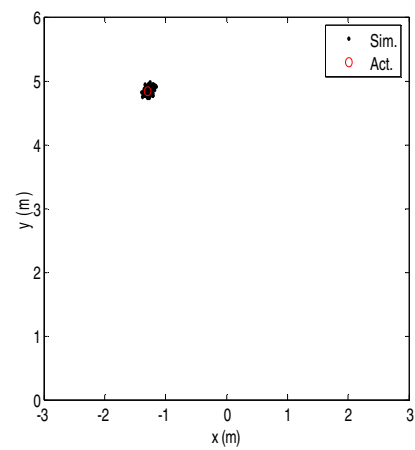

(a) $\theta_{2}=40^{\circ}$

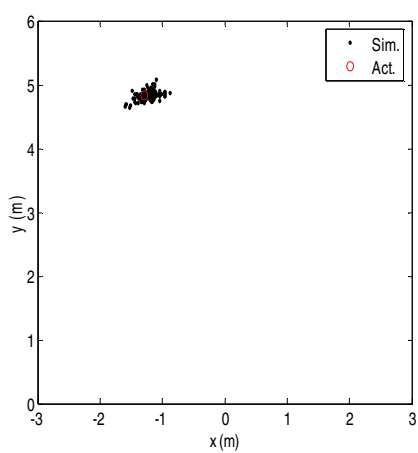

(b) $\theta_{2}=10^{\circ}$
Figure 4. Estimated target positions using frequency set I with different DOA of the reflection path.

\section{CONCLUSION}

We have investigated the problem of locating a target in a multipath propagation environment by using a multifrequency array. The proposed method effectively utilizes a small number of spatial and spectral degrees-of-freedom to decorrelate the coherent paths and perform DOA estimation, spatial filtering, and range estimation, yielding robust target localization. Simulation results demonstrated the effectiveness of the proposed method and examined the effects of various parameters to its performance.

\section{REFERENCES}

[1] F. Ahmad, M. Amin, and P. Setlur, "Through-the-wall target localization using dual-frequency CW radars," in Proc. SPIE, vol. 6201, Orlando, FL, April 2006.

[2] F. Ahmad, M. G. Amin, and P. D. Zemany, "Dual-frequency radars for target localization in urban setting," IEEE Trans. Aerospace and Electronic Systems, vol. 45, no. 4, pp. 1598-1609, Oct. 2009.

[3] Y. Zhang, M. G. Amin, and F. Ahmad, "Time-frequency analysis for the localization of multiple moving targets using dualfrequency radars," IEEE Signal Processing Letters, vol. 15, pp. 777-780, 2008.

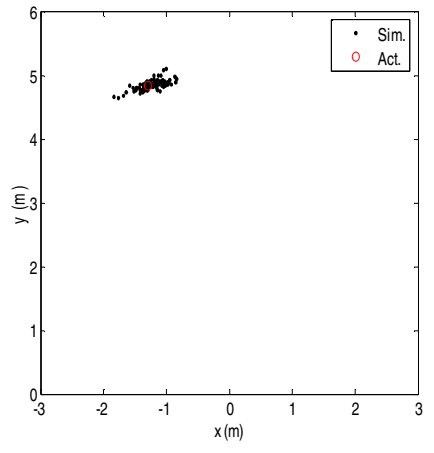

(a) $R_{2}=6 \mathrm{~m}$

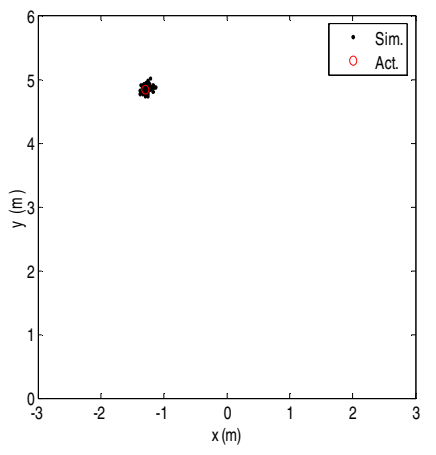

(b) $R_{2}=8 \mathrm{~m}$
Figure 5. Estimated target positions using frequency set I with different length of the reflection path.

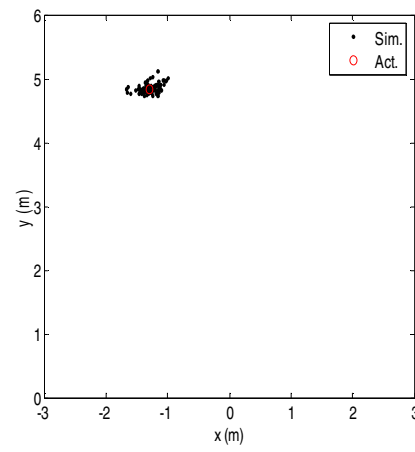

(a) $h_{2}=0.3 e^{j \pi / 4}$

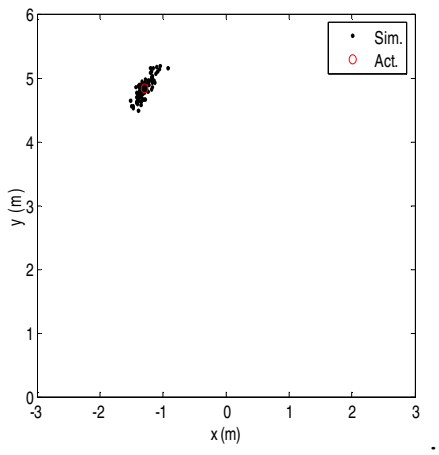

(b) $h_{2}=0.7 e^{-j \pi / 4}$
Figure 6. Estimated target positions using frequency set I with different reflection coefficient of the reflection path.

[4] A. Lin and H. Ling, "Three-dimensional tracking of humans using very low-complexity radar," Electronics Letters, vol. 42, no. 18, pp. 10621064, Aug. 2006.

[5] X.-G. Xia and G. Wang, "Phase unwrapping and a robust Chinese remainder theorem," IEEE Signal Processing Letters, vol. 14, no. 4, pp. 247-250, April 2007.

[6] X. Li, Y. Zhang, and M. G. Amin, "Multifrequency-based range estimation of RFID tags," in Proc. IEEE Int. Conf. on RFID, Orlando, FL, April 2009.

[7] Y. Zhang, X. Li, and M. G. Amin, "Array processing for RFID tag localization exploiting multi-frequency signals," in Proc. SPIE Wireless Sensing and Processing Conf., Orlando, FL, April 2009.

[8] T.-J. Shan, M Wax, and T Kailath, "On spatial smoothing for directionof-arrival estimation of coherent signals," IEEE Trans. Acoust., Speech, Signal Proc., vol. ASSP-33, pp. 806-811, Aug. 1985.

[9] H. Wang and M. Kaveh, "Coherent signal-subspace processing for the detection and estimation of angles of arrival of multiple wide-band sources," IEEE Trans. Acoust., Speech, Signal Process., vol. ASSP-33, no. 4, pp. 823-831, Aug. 1985.

[10] H. Hung and M. Kaveh, "Focussing matrices for coherent signalsubspace processing," IEEE Trans. Acoust. Speech Signal Process., vol. 36, no. 8, pp. 1272-1281, Aug. 1988.

[11] M. A. Doron and A. J. Weiss, "On focusing matrices for wideband array processing," IEEE Trans. Signal Process., vol. 40m no. 6, pp. 1295-1302, June 1992. 